\title{
THE PLASMA WINDOW: A WINDOWLESS HIGH PRESSURE-VACUUM INTERFACE FOR VARIOUS ACCELERATOR APPLICATIONS*
}

\author{
A.I. Hershcovitch, E.D. Johnson, Brookhaven National Laboratory, Upton, NY 11973
}

\author{
R.C. Lanza, Massachusetts Institute of Technology, Cambridge, MA 02139
}

\begin{abstract}
The Plasma Window is a stabilized plasma arc used as an interface between accelerator vacuum and pressurized targets. There is no solid material introduced into the beam and thus it is also capable of transmitting particle beams and electromagnetic radiation with low loss and of sustaining high beam currents without damage. Measurements on a prototype system with a $3 \mathrm{~mm}$ diameter opening have shown that pressure differences of more than 2.5 atmospheres can be sustained with an input pressure of 10-6 Torr. The system is capable of scaling to higher-pressure differences and larger apertures. Various plasma window applications for synchrotron light sources, high power lasers, internal targets, high current accelerators such as the HAWK, ATW, APT, DARHT, spallation sources, as well as for a number of commercial applications, will be discussed.
\end{abstract}

\section{INTRODUCTION}

Many industrial and scientific processes like ion material modification, electron beam melting and welding, as well as generation of high energy radiation are performed exclusively in vacuum nowadays, since electron guns, ion guns, their extractors and accelerators must be kept at a reasonably high vacuum. Consequently, there are numerous limitations, among which are low production rates due to required pumping time, limits on the size of target objects, and degradation of particle beams and radiation through foils or differentially pumped sections.

For transmission of high-energy synchrotron radiation thin beryllium windows are used (or SiN in a few cases). Attenuation and spatial structure non-uniformities, which attend the use of these conventional windows, represent a significant problem for various applications in synchrotron radiation research. Most of the problems are the result of window heating and degradation of the radiation. The target systems used for accelerator based neutron sources greatly limits the current, energy, and energy focus of the neutron beam. A pressurized

*Work supported in part by the U.S. Department of Energy under Contract No. DE-AC02-76CH00O16

Hershcovitch@bnldag.bnl.gov windowless gas target system removes these limitations by removing all physical boundaries between the accelerator and the gas target. The gas target itself poses no limitations on the beam since it can be constantly cooled and replenished.

A novel apparatus, which utilized a short plasma arc, was successfully used to provide a vacuum-atmosphere interface as an alternative to differential pumping, and an electron beam was successfully propagated from vacuum to atmospheric pressure. [1,2]

Windowless gas targets, for neutron production, have been constructed using differentially pumped gas systems with a series of rotating valves. Although these systems have demonstrated the feasibility of such techniques, they are limited to use with pulsed accelerators with relatively low duty cycles, around $2.5 \%$.[3]

Such a windowless gas target system can be formed by creating a plasma discharge between the highpressure target chamber and the low-pressure vacuum chamber. The high temperature of the plasma discharge $\left(12,000^{\circ} \mathrm{K}\right.$ at the edges and $15,000^{\circ} \mathrm{K}$ at the center $)$ causes the plasma to be over 40 times less dense than the target gas while being more viscous. These two properties combine to form an opaque plug. This plug allows the accelerated particles, or radiation to pass through while greatly reducing any flow between the high pressure and low pressure chambers. In the best results to date[4] of such a system, 40 psia were supported in the target chamber with 0.032 Torr in the first vacuum chamber.

The theory, experimental status, and possible applications of the Plasma Window will be discussed in this report.

\section{PRINCIPLE OF OPERATION}

Plasmas can be used for vacuum separation, interface with atmosphere, and as lenses. Ideally, maximizing the plasma temperature would optimize the first two applications, while maximizing the discharge current should broaden the use of the latter. For this Plasma Window, a "wall stabilized discharge" is used, i.e., a 
stable plasma discharge is created down the center of a cooled tube. The temperature of the plasma ranges from $12,000^{\circ} \mathrm{K}$ at the edges to $15,000^{\circ} \mathrm{K}$ in the center. These temperatures are sufficiently low enough so that the plasma flow is dominated by intermolecular collisions (Knudsen number much less then one). This allows the plasma to be treated as a high temperature gas.

By treating the plasma as a high temperature gas, the ideal gas equation can be applied

$$
P=\rho \cdot R \cdot T
$$

where $P$ is the pressure, $T$ is the temperature, $\rho$ is the density, and $R$ is the ideal gas constant.

Using this equation to compare the density of the plasma arc at $12,000^{\circ} \mathrm{K}$ to the density of the gas in the target chamber at $300^{\circ} \mathrm{K}$, it is clear that the plasma is 40 times less dense while at the same pressure. This low density at high pressures is key in allowing the plasma to support large pressure differentials while still being transparent to the accelerated beam.

The pressure differential that the plasma arc will be able to support is determined by the flow rate of the plasma. This flow rate of an ideal gas through a small thin tube can be given by:

$$
\mathrm{Q}=\frac{\pi \cdot \mathrm{R}^{4}}{8 \cdot \mu} \cdot\left(\frac{\mathrm{dp}}{\mathrm{dx}}\right)
$$

where $Q$ is the flow rate, $\mu$ is the viscosity, $R$ is the ideal gas constant, and $d p / d x$ is the pressure difference in pipe. In order to minimize the flow through the pipe while maximizing the pressure differential, it is desirable to increase the viscosity as much as possible. Typical fluid mechanics shows that viscosity increases with temperature.

As the plasma gas is raised in temperature, the viscosity becomes directly proportional to the temperature at higher powers.[1.5] It is important to note that this plasma is in the absence of any applied magnetic field.

An added benefit gained from the use of the plasma window is a focusing effect on charged particle beams. As current flows in the plasma arc it creates a circular magnetic field around the center of the plasma which results in a field in the $\theta$ direction. With proper arc current orientation, charged particles passing through the plasma interact with the magnetic field and are subjected to an inward force given by

$$
F=q \cdot \vec{v} \times \bar{B}
$$

where $q$ is the charge of the particle, $v$ is its velocity, and $B$ is the induced magnetic field. More details on the theory of operation can be found in references 1 and 2 .

\section{EXPERIMENTAL RESULTS}

Various plasma window experimental results can be found in references 1,2, and 4. The plasma window utilizes an arc discharge. The arc is a wall-stabilized type cascade arc discharge. The arc has been operated in argon and in helium.

\subsection{Previous Experimental Results}

The best results to date have been the following:

- Differential pumping: vacuum-atmosphere separation across a $2.36 \mathrm{~mm}$ diameter $4 \mathrm{~cm}$ long arc was achieved. With the arc open to atmosphere, pressure in a 4" pipe, on which plasma window was mounted. was 0.032 Torr. Gain over conventional differential pumping, by comparing pressures with plasma arc on versus plasma arc off, yielded a factor of $10^{4}$ improvement. In that particular experiment[4] the vacuum system baseline pressure of $10^{-6}$ Torr was reached 20" from the plasma window (which was opened to atmosphere).

- High pressure gas cells were successfully maintained: 40 psia was separated from accelerator vacuum (for application to neutron generation); a 72 psia gas was separated from atmosphere, and a 5 $\mathrm{mW}$ He-Ne laser beam was passed through it (for gas laser applications).

- A $175 \mathrm{KeV}$ electron beam was transmitted from vacuum through the plasma window to atmospheric pressure.

- Compatibility tests for transmission of electromagnetic radiation indicated that the plasma window does not generate electromagnetic interference, and that $\mathrm{X}$-rays (away from resonance) are transtrmitted with negligible attenuation.

\subsection{Possible Improvements}

Testing of the Plasma Window system has not only proven the validity of the windowless vacuum-pressure interface, but has highlighted many areas for improvements.

Cooling of the chamber next to the plasma window in the most apparent area for improvement. Overheating of the system not only risks damaging equipment but also increases the rate of outgassing and thus increases the overall pressure in the system.

A dramatic decrease $[1,2,4]$ in the flow from the first vacuum chamber to the second vacuum chamber can, in principle (based on density and temperature profiles of similar arcs), be gained by adding skimmers to the first chamber. These internally cooled curved blades will serve to deflect the gas streaming from the cool annulus of the plasma window, as well as any gas which is not directly on the beam line. Aperture size in the Plasma Window can be increased in order to accommodate 
larger beam diameters. If gas flow through the Plasma Window is dominated by leak through the cool annulus, reasonable increases in window diameter will marginally increase the flow rate through the snimmer system.

\section{APPLICATIONS}

Several different types of accelerator systems can benefit from the utilization of the Plasma Window system.

\subsection{Commercial Applications}

Non-vacuum electron beam welding: With plasma windows, higher production rates, no limit on the size of target objects, and high quality electron beams in atmosphere.

Non-vacuum material modifications by ion implantation, and dry etching, or micro-fabrication: Presently performed only in vacuum, since ion beams at energies used in these applications are completely attenuated by foils and by long differentially pumped sections. Potentially very large yet unexplored market.

Electron beam melting: for manufacturing alloys is performed at a pressure of about $10^{-2}$ Torr. A major drawback of operating at this pressure range is the loss of elements with low vapor pressure. Consequently, it is desirable to raise the operating pressure to a higher level. Interested company.

Electron beam generation of photo-neutrons for the production of medical isotopes (e.g., Tc-99): A $40 \mathrm{MeV}$ electron beam strikes a $\mathrm{W}$ target. Resultant radiation can dislodge a neutron (via a giant resonance) to create a new element. With a plasma window, the target can be in the air, sufficiently cooled to absorb an intense electron beam to generate photo-neutrons.

Windowless gas targets for fast neutron radiography to detect nitrogen (weapons) and carbon (diamonds), as well as for other forms of neutron tomography and therapy (BNCT).

Windows for high power lasers (especially highpressure gas lasers).

\subsection{Scientific Applications}

Windowless beamlines for transmission of synchrotron radiation: Plasma windows offer many advantages over presently used beryllium windows: radiation passes through the window unaffected. A plasma window cannot be damaged by radiation. UV filter for rejection of 'high-order' light is of significant benefit to experiments like threshold photoionization spectroscopy, where contamination even at the $10^{-1}$ harmonic content can obscure the features of the spectrum of interest. X-ray microscopies, since it is free from the attenuation and spatial structure that attend the use of conventional window materials (e.g. beryllium or SiN), i.e., no scratches.

Windowless gas targets for fast (fusion) neutron generation (neutron sources).

Radioactive waste transmutation (ATW): protons are accelerated to $2 \mathrm{GeV}$ unto a heavy metal target in air. Resultant spallation neutrons reduce radioactive waste. Presently, windows limit proton output.

APT. tritium production by accelerator: Solid windows limit beam and tritium output.

Spallation Neutron Source (SNS): To replace various solid windows with cooling problem.

Windows for high power $x$-ray source (DARHT).

Internal (gas or plasma) targets, strippers, lenses in storage rings: A plasma stripper/lens or an internal gas target "sandwiched" between two plasma windows. Examples: BNCT based on recirculating proton beams and various internal targets (including spin polarized).

Fast acting valves in UHV beamlines. In case of vacuum breach, plasmas can be ignited faster than mechanical valves without damage to beamline (unlike, presently used, msec spring loaded shutters).

\section{CONCLUSIONS}

This examination of the Plasma Window has shown the viability of using a plasma arc to support a large pressure differential. which could be further improved. It operates steady state without maintenance for 2000 hours.

Through the use of the Plasma Window system, limitations on beam current, energy, energy focus and others have been removed. This allows the Plasma Window system to enhance a wide rangc of applications, which were not listed like using the HAWK intense electron beam to heat gas in hypersonic wind tunnels.

\section{REFERENCES}

1.Hershcovitch, Ady. "High-pressure arcs as vacuum-atmosphere interface and plasma lens for nonvacuum electron beam welding machines, electron beam melting, and nonvacuum ion material modification." Journal of Applied Physics. Vol. 78 (9), November, 1995

2. Hershcovitch. Ady. "A Plasma Window for Transmission of Particle Beams and Radiation from Vacuum to Atmosphere for Various Applications." Physics of Plasmas, vol.5, no.5, May 1998, pp.21306.

3.E.B. Iverson, R.C. Lanza, and L.M.Lidsky, "A Windowless Gas Target Neutron Source", 5th Intemational Conference on Applications of Nuclear Techniques, Crete, 1996 in Proceedings of SPIE - the International Society for Optical Engineering, vol.2867, 1997, pp.513-16.

4. W. Gerber, R.C. Lanza, A. Hershcovitch, P. Stefan, C. Castle. E. Johnson, Invited Talk on DThe Plasma Porthole: a Windowless Vacuum-Pressure Interface With Various Accelerator Applications] at 15 th International Conference on the Application of Accelerators. in Research and Industry CAAR I'98, Denton, Texas, November $4-7$, 1998; to be published in RSI.

5.Lontovich, Acad., Reviews of Plasma Physics, Volume 1. Consultants Bureau, New York, 1969. 